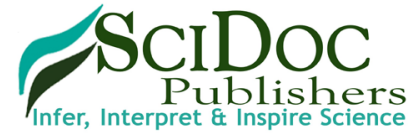

International Journal of Vaccines and Research (IJVR)

ISSN 2572-7427

\title{
A Comparative Approach on Shelf Life Stability of Tetanus Toxoid Vaccine Produced from Imported and Locally Formulated Bulk in Private Sector Facility of Pakistan
}

Review Article

Hassan A K ${ }^{1 *}$, Kulsum A², Hassan A U

Amson Vaccines and Pharma (Pvt.) LTD, Islamabad.

Abstract

Tetanus is an acute ailment, pictured by the substantial increase in rigidity and spastic cramps in striated muscle. The substandard tetanus toxoid vaccine might increase the incidence of neonatal tetanus. WHO has taken many steps to help national regulatory authorities and vaccine manufacturers to make sure its quality and efficacy. It has developed international principles of stability analysis of every vaccine that is available in the form of recommendations and guidelines. The aim of the present study was to ensure the efficacy of tetanus vaccine produced by manufacturer of private sector (Amson Vaccine and Pharma (Pvt) LTD. Islamabad, Pakistan). All stability studies performed on final products stored at $2-8^{\circ} \mathrm{C}$ and evaluated at near expiry and even three months after its expiry month. The study gives efficacy comparison between Tetanus vaccine injection filled from imported RTF Bulk and Formulated Tetanus Toxoid Bulk from the private sector. The findings indicate that the formulated tetanus vaccine is equally effective when checked its safety profile in live animals. The study also reveals that the product remains stable till last day of its expiry month provided if the cold chain was maintained between $2-8^{\circ} \mathrm{C}$ till consumed.

Keywords: Shelf Life Studies; Tetanus Toxoid; Pakistan.

\section{Introduction}

Tetanus is an acute, typically pernicious illness that is characterized by sweeping increased compactness and spastic spasms of skeletal muscles [1]. It remains a significant reason behind death in developing countries. In 2008, more than 59,000 deaths were caused by neonatal tetanus, accounting for $1 \%$ of international death rate, 1 st and foremost in establishing nations and is caused by tetanus-pasmin, a neurotoxin fashioned by gram positive bacterium clostridium tetani [2]. Neonatal tetanus remains a community pathological state in several developing countries together with Pakistan, wherever it's one in all the principal causes of infant and infant death. In pakistan, infant tetanus accounts for $18-38 \%$ and $17-22 \%$ of all infant and child deaths [3, 4]. WHO estimates that in 2013 (the latest year for which estimates are available), 49,000 newborns died from NT, a 94\% reduction from the situation in the late 1980s [5]. In Pakistan, 898 cases of Neonatal tetanus reported till july 2014 [6]. Vaccination is the most potent methodology against this illness and has gradual impact to subside mortalities [7]. Tetanus vaccine is given to neonates and a pregnant lady, as in mid 1970s it had been enclosed in WHO's expanded program of immunization [8]. Consistence of vaccine contains a paramount execution within the distinction of immunization programs worldwide [9]. The poor quality tetanus toxoid vaccine will steer towards the accession within the prevalence of neonatal tetanus.

Efforts of World Health Organization in reinforcement of national regulatory authorities along with vaccine manufacturers in maintaining the quality of vaccines for immunization is promising. Recommendations and guidelines are available that are applied to the production and quality control of vaccines. As per WHO recommendations stability study is the most critical evaluation of quality assessment. More emphasis is laid upon the shelf life stability to ensure quality, safety and efficacy of vaccines [10]. In Pakistan, Amson Vaccines and Pharma (Pvt) Limited, Islamabad is producing tetanus vaccine by formulation process, filling and packaging from imported tetanus concentrate of tetanus toxoid. The comparative study of Formulated and RTF bulk filled tetanus vaccine was carried out on five batches of each bulk filled tetanus toxoid vaccines. i.e. 510 - 514 tetanus vaccine filled from RTF bulk and 568 - 572 tetanus vaccine filled from

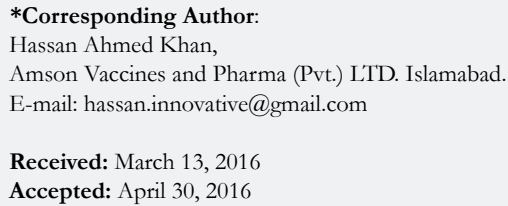

Citation: Hassan A K, Kulsum A, Hassan A U (2016) A comparative approach on shelf life stability of tetanus toxoid vaccine produced from imported and locally formulated bulk in private sector facility of Pakistan. Int J Vaccines Res. 3(2), 11-18. doi: http://dx.doi.org/10.19070/2572-7427-160003

Copyright: Hassan A $\mathbf{K}^{\circ}$ 2016. This is an open-access article distributed under the terms of the Creative Commons Attribution License, which permits unrestricted use, distribution and reproduction in any medium, provided the original author and source are credited. 
Formulated bulk. The filling date of RTF bulk filled batches was Dec. 2010 and expiry date was Nov. 2013. The filling date of formulated bulk filled batches was Jan, 2012 and expiry date was Dec, 2014. Vaccines from all these batches were checked at storage conditions $\left(2-8^{\circ} \mathrm{C}\right)$ for purpose of real shelf life stability studies. The aim of these studies was to ensure the quality, potency and efficacy of tetanus vaccines produced by pioneer manufacturer in the private sector of Pakistan and to ensure stability of tetanus toxoid at $2-8^{\circ} \mathrm{C}$ storage/transportation cold chain temperature. This study gave a comparison between tetnaus toxoid vaccine produced from imported RTF bulk and from formulated bulk of private sector with real time shelf life stability studies resulting in the wider scope of this article.

\section{Difference in RTF \& Formulated Tetanus Toxoid Bulk}

\section{Ready to Fill (RTF) Tetanus Toxoid Bulk}

Ready to fill bulk is imported in 20Lit. Nelgene bottles by maintaining the refrigeration temperature $2-8^{\circ} \mathrm{C}$, conforming by shipment temperature loggers provided with each bulk bottle by the vendor. All the bulk bottles are carefully checked for any mishandling and stored in cold room at $2-8^{\circ} \mathrm{C}$. Each bulk bottles is filled in sterile close mouth glass ampoules with separate batch ID under strict aseptic conditions without any change in formulation. Filled ampoules are stacked in cold room at $2-8^{\circ} \mathrm{C}$, packed and marketed on final release.

\section{Local Formulated Tetanus Toxoid Bulk}

In case of local formulation, tetanus toxoid (Antigen) concentrate is only imported, a quality Aluminum Phosphate gel is prepared at Amson Vaccine \& Pharma injectable formulation facility using different quality chemicals. Antigen concentrate is diluted as per requirement and adsorbed on the surface of in-house prepared Aluminum Phosphate gel under controlled parameters of temperature, humidity, $\mathrm{pH}$ of the solution, rotation per minutes and process duration, during Tetanus toxoid bulk preparation. Inprocess check and quality of bulk is assured at different stages. After tetanus toxoid bulk formulation, bulk bottles are stored in cold room at $2-8^{\circ} \mathrm{C}$. Each bulk bottle is filled in sterile close mouth glass ampoules with separate batch identification under strict aseptic conditions. Filled ampoules are stacked in cold room at $2-8^{\circ} \mathrm{C}$, packed and marketed on final release.

\section{Materials and method}

\section{Study design}

A total of ten filled batches, five from each bulk (RTF \& Formulated) production were tested. Real time Stability studies determining efficacy was performed on final products stored at $2-8^{\circ} \mathrm{C}$.

- Imatet filled batches results 06 month before expiry date.

- Imatet filled batches results 03 month before expiry date.

- Imatet filled batches results at expiry month.
- Imatet filled batches results 03 month after expiry.

The following parameters were tested to check the efficacy of the two bulk filled product during stability study: InVivo Potency, Sterility test, Abnormal Toxicity, and physicochemical parameters i.e Antigen content. $\mathrm{Al}^{+++}$content, Free Formaldehyde content, Thiomersal content and $\mathrm{pH}$ for tetanus toxoid vaccine (BLG/ UNDP/77-2Rev.1).

\section{Mice Challenge InVivo Potency}

Mice Challenge InVivo Potency is performed by mice immunization and Tetanus Toxin challenge method according to (BLG/UNDP/77-2 Rev.1). InVivo potency test of all RTF \& Formulated filled batches were tested at animal house facility of Amson Vaccines \& Pharma (Pvt) Ltd., Islamabad.

Requirement: BALB-c strain of Swiss albino mice, Sterile Normal saline, Pipettes $5 \mathrm{ml}, 10 \mathrm{ml}, 100 \mathrm{ml}$ Flask, and sterile disposable syringes. Reference vaccine. Three dilutions of test as well as reference vaccines are prepared having 0.020, 0.008 and 0.0032 $\mathrm{ml}$ vaccine/dose. The dose of $0.5 \mathrm{ml}$ is injected in subcutaneous route on dorsal view of hind limb in a group of 18 mice individually. After 28-30 days, each immunized mouse is challenge with tetanus toxin of $50 \mathrm{LD}_{50} /$ dose.

Interpretation of result: Note the death and survival of mice and calculate the potency in term of IU/dose by probit analysis method of WHO (BLG/UNDP/77-2 Rev.1).

Acceptance Criteria: InVivo potency of tetanus toxoid vaccine must not be less than $40 \mathrm{IU} /$ dose.

\section{Sterility test}

Performed for every sterile component of product as well as on final containers.

Requirement: Sterile and Pre incubated $35 \mathrm{ml}$ Fluid Thioglycollate Medium Tubes., Incubators (one is set at $20-25^{\circ} \mathrm{C}$ and one is set at $30-35^{\circ} \mathrm{C}$ ), Disposable Syringes, Pre-incubated TSA (Tryptone Soya Agar) plates. Mark the one set of the tubes as media control, one set as Test and one set as Syringe Control. The test is performed by direct inoculation of sample in Fluid Thioglycollate medium (for aerobic \& anaerobic growth) and incubated at $30-35^{\circ} \mathrm{C}$ and other set of Thioglycollate medium incubated at $20-25^{\circ} \mathrm{C}$ for 14 days.

Interpretation of result: Absence of any type of growth in the form of turbidity after 14 days incubation of above mentioned media indicates that the samples are sterile.

Acceptance Criteria: The product should comply the sterility test and declared as sterile.

\section{Abnormal Toxicity}

Each final filled batch of vaccine is tested intra peritoneal injection in mice and Guinea pigs. Two Guinea pigs and five mice are injected for each batch, Inject Single human dose or maximum $1 \mathrm{ml}$ in mice and five human doses or maximum $5 \mathrm{ml}$ in each guinea pig. 
Interpretation of result: The animals are observed for seven days for any abnormality or toxic reaction (Skin rash) including loss in weight or death of any animal.

Acceptance Criteria: The product under investigation must be non toxic.

\section{Physicochemical parameters}

Antigen content: Antigen content determination is performed according to WHO guideline on each bulk lot as well as final batch. This test is performed by Ramon titration method to identify the antigen content in the product, the reaction is called flocculation. The antigen content is expressed either Lf/ $\mathrm{ml}$ or Lf/ dose. This is immunological antigen antibody binding test.

Antigenic strength and the purity of tetanus toxoid, prior to its use in the production of vaccine are determined by a flocculation test (to express Lf unit in toxoid). The WHO provides calibrated tetanus toxoid as a primary international standard to help in standardization of assays used to determine the Lf unit of toxoid. It is also used as an antigen for independent InVitro quality control assessments of vaccines and anti-tetanus preparations.

Requirement: Reference Anti-tetanus Serum (equine) 100Lf/ml (ATS), Normal saline (sterile), Water bath set at $50^{\circ} \mathrm{C}$, micropipette (100-100ul), Sodium Citrate. Make the dilution of tetanus toxoid with Normal Saline then mixes with required quantity of ATS incubate in water bath at $50^{\circ} \mathrm{C}$.

Interpretation of result: Flocculation reaction occurs within one hour observe the reaction in black back ground; note the tube which first flocculates. Calculate the $\mathrm{Lf} / \mathrm{ml}$ by the following formula: $\mathrm{Lf} / \mathrm{ml}=$ Amount of antitoxin in $\mathrm{ml} \mathrm{x} \mathrm{Lf} / \mathrm{ml}$ of antitoxin/ amount of test sample.

Acceptance Criteria: The limit of flocculation must lie between 5-25 Lf/dose.

\section{$\mathrm{Al}^{+++}$content determination}

Titration Method

\section{Requirement:}

\section{Reagents Used}

Sulphuric Acid, Nitric Acid, Glacial acetic acid, Sodium acetate, Methyl orange, Pyridilazonaphtol (Indicator) (PAN), $0.02 \mathrm{M}$ EDTA Solution, CuSO4.5H2O (VS) Solution, Sodium Hydroxide, Ammonium Acetate Buffer ( $\mathrm{pH}$ 4.4), Pyridylazonaphthol (Pan) $(0.1 \% \mathrm{w} / \mathrm{v}), 0.02 \mathrm{M}$ Disodium Edetate.

Calculation of Results: a =mean consumption of $0.02 \mathrm{M} \mathrm{CuSO} 4$ for the black, $b=$ mean consumption for the sample, $0.5396=$ quantity of $\mathrm{Al}(\mathrm{mg})$, which is equivalent to $1 \mathrm{ml}$ of $0.02 \mathrm{M}$ disodium edetate, $\mathrm{V}=$ volume of the sample $[(\mathrm{a}-\mathrm{b}) \times 0.5396] / \mathrm{V}=\mathrm{mg}$ of $\mathrm{Al}$ per ml sample.

Acceptance Criteria: Aluminium content of tetanus toxoid must not exceed $1.25 \mathrm{mg} / \mathrm{ml}$.

\section{Thiomersal content determination}

Requirement: For the determination of thiomersal content, following materials are required: Thimerosal (0.01\%) Solution Standard, 0.001\% Dithizone Reagent, Concentrated Nitric acid, 1\% Ammonium Acetate Solution, Chloroform, Tap water, Separating funnel, Spectrophotometer, Beaker.

Calculation of Results: Thiomersal Content $=$ Absorbance of std. x Conc. Of standard / Absorbance of sample.

Acceptance Criteria: Thiomersal content should lie between $0.085 \%$ to $0.115 \%$.

\section{PH determination}

$\mathrm{PH}$ of tetanus toxoid final containers sample should be between 6.0 to 7.0 .

\section{Results}

\section{Stability data of imported Ready to Fill (RTF) bulk}

Stability data of imported Ready to Fill (RTF) bulk filled batches under conventional shelf life circumstances from $2-8^{\circ} \mathrm{C}$ is evaluated. Consequences for each test have been given after 03 months for individual clutch of vaccines expiry before 06 months. The antigenic composition perpetuated its congregation and found to be $20 \mathrm{Lf} / \mathrm{ml}$. Potency data emulates that applicable vigor of vaccines for the entire period of time was perpetuated and after 39 months found to be $(62,62,62,62$ and 66) IU/dose for 1, 2, 3, 4 and 5 th batch respectively. Other safety and physical manifestation of vaccines are agreeable. Free formaldehyde content, $\mathrm{Al}^{+++}$content, thiomersal concentration and $\mathrm{pH}$ of vaccines are well within the ascertained range for the entire period of time are found also illustrated by data. Free formaldehyde content after 39 months come up within limit of less than $0.02 \%$. Al ${ }^{+++}$content is reported $0.324 \mathrm{mg} /$ dose, $0.270 \mathrm{mg} /$ dose, $0.270 \mathrm{mg} /$ dose, $0.270 \mathrm{mg} /$ dose, $0.297 \mathrm{mg} /$ dose for batch $1,2,3,4$ and 5 respectively. No significant change in $\mathrm{pH}$ is observed in respective batches after 39 months but still fall well within limits i.e, 6 -7. Sterility, Antigenic content, Bacterial Endotoxin along with safety profile meets the WHO standards. It can be concluded that vaccine prevail its efficacy and safe for human use.

\section{Stability data of local formulated bulk}

Stability data of local formulated bulk filled congregated from four distinctive clutches of vaccines deliberated under conventional shelf life circumstances from $2-8^{\circ} \mathrm{C}$ for 39 months is evaluated. Consequences for each test have been given after 03 months for individual clutch of vaccines expiry before 06 months. The antigenic composition perpetuated its congregation and found to be $20 \mathrm{Lf} / \mathrm{ml}$. Potential or immunogenicity data emulates that applicable vigor of vaccines for the entire period of time was perpetuated and after 39 months found to be (58, 57, 55, 57 and 61) IU/dose for 1, 2, 3, 4 and 5th batch respectively. Other safety and physical manifestation of vaccines are agreeable. Free formaldehyde content, $\mathrm{Al}^{+++}$content, thiomersal concentration and $\mathrm{pH}$ of vaccines are well within the ascertained range for the entire period of time are found also illustrated by data. Free 
Table 1. Initial Test Data of Ready to Fill (RTF) TT Bulk filled Batches.

\begin{tabular}{|c|c|c|c|c|c|c|c|c|c|}
\hline \multirow[t]{2}{*}{ B\# } & \multirow[t]{2}{*}{$\mathrm{pH}$} & \multirow[t]{2}{*}{$\mathrm{Al}^{+++}$Content } & \multirow{2}{*}{$\begin{array}{c}\text { Formaldehyde } \\
\text { Content }\end{array}$} & \multirow[t]{2}{*}{ Thiomersal } & \multirow[t]{2}{*}{ Sterility Test } & Ab. & \multirow{2}{*}{$\begin{array}{l}\text { Antigen Con- } \\
\text { tent }\end{array}$} & \multirow{2}{*}{$\begin{array}{c}\text { Bacterial } \\
\text { Endotoxin }\end{array}$} & \multirow[t]{2}{*}{ In Vivo Potency } \\
\hline & & & & & & Toxicity & & & \\
\hline 510 & 6.32 & $0.324 \mathrm{mg} /$ dose & LT $0.02 \%$ & $0.096 \%$ & Pass & Pass & $10 \mathrm{Lf} /$ Dose & $0.198 \mathrm{EU} / \mathrm{ml}$ & 86.31 IU/Dose \\
\hline 511 & 6.26 & $0.270 \mathrm{mg} /$ dose & LT $0.02 \%$ & $0.096 \%$ & Pass & Pass & $10 \mathrm{Lf} /$ Dose & $0.215 \mathrm{EU} / \mathrm{ml}$ & $90.61 \mathrm{IU} /$ Dose \\
\hline 512 & 6.29 & $0.270 \mathrm{mg} /$ dose & LT $0.02 \%$ & $0.097 \%$ & Pass & Pass & $10 \mathrm{Lf} /$ Dose & $0.230 \mathrm{EU} / \mathrm{ml}$ & $90.61 \mathrm{IU} /$ Dose \\
\hline 513 & 6.28 & $0.270 \mathrm{mg} /$ dose & LT $0.02 \%$ & $0.098 \%$ & Pass & Pass & $10 \mathrm{Lf} /$ Dose & $0.235 \mathrm{EU} / \mathrm{ml}$ & $90.61 \mathrm{IU} /$ Dose \\
\hline 514 & 6.31 & $0.297 \mathrm{mg} / \mathrm{dose}$ & LT $0.02 \%$ & $0.0101 \%$ & Pass & Pass & $10 \mathrm{Lf} /$ Dose & $0.170 \mathrm{EU} / \mathrm{ml}$ & $90.85 \mathrm{IU} /$ Dose \\
\hline Specs. & $6-7$ & NMT $1.25 \mathrm{mg} /$ dose & NMT $0.02 \%$ & $\begin{array}{c}0.0085 \% \text { - } \\
0.0115 \%\end{array}$ & Sterile & Non-toxic & 5-25 Lf / dose & NMT $25 \mathrm{Eu} / \mathrm{ml}$ & NLT 40 IU/Dose \\
\hline
\end{tabular}

Manuf. Date; Dec. 2010

Expiry Date; Nov. 2013

Table 2. Stability Data of Different RTF Bulk Filled TT Batches (06 month Before Expiry, 30 Months).

\begin{tabular}{|c|c|c|c|c|c|c|c|c|c|}
\hline B\# & $\mathbf{p H}$ & $\mathbf{A l}^{++}$Content & $\begin{array}{c}\text { Formaldehyde } \\
\text { Content }\end{array}$ & Thiomersal & $\begin{array}{c}\text { Sterility } \\
\text { Test }\end{array}$ & $\begin{array}{c}\text { Ab. } \\
\text { Toxicity }\end{array}$ & $\begin{array}{c}\text { Antigen } \\
\text { Content }\end{array}$ & $\begin{array}{c}\text { Bacterial } \\
\text { Endotoxin }\end{array}$ & $\begin{array}{c}\text { InVivo } \\
\text { Potency }\end{array}$ \\
\hline 510 & 6.60 & $0.243 \mathrm{mg} /$ dose & LT $0.02 \%$ & $0.0092 \%$ & Pass & Pass & $10 \mathrm{Lf} /$ Dose & $0.193 \mathrm{EU} / \mathrm{ml}$ & $75.28 \mathrm{IU} / \mathrm{Dose}$ \\
\hline 511 & 6.57 & $0.296 \mathrm{mg} /$ dose & LT $0.02 \%$ & $0.0093 \%$ & Pass & Pass & $10 \mathrm{Lf} /$ Dose & $0.210 \mathrm{EU} / \mathrm{ml}$ & $70.64 \mathrm{IU} / \mathrm{Dose}$ \\
\hline 512 & 6.55 & $0.269 \mathrm{mg} /$ dose & LT $0.02 \%$ & $0.0092 \%$ & Pass & Pass & $10 \mathrm{Lf} /$ Dose & $0.230 \mathrm{EU} / \mathrm{ml}$ & $70.87 \mathrm{IU} / \mathrm{Dose}$ \\
\hline 513 & 6.47 & $0.269 \mathrm{mg} /$ dose & LT $0.02 \%$ & $0.0094 \%$ & Pass & Pass & $10 \mathrm{Lf} /$ Dose & $0.230 \mathrm{EU} / \mathrm{ml}$ & $75.13 \mathrm{IU} / \mathrm{Dose} /$ \\
\hline 514 & 6.35 & $0.296 \mathrm{mg} /$ dose & LT $0.02 \%$ & $0.0092 \%$ & Pass & Pass & $10 \mathrm{Lf} /$ Dose & $0.175 \mathrm{EU} / \mathrm{ml}$ & $75.13 \mathrm{IU} / \mathrm{Dose}$ \\
\hline
\end{tabular}

Table 3. Stability Data of Different RTF Bulk Filled TT Batches (03 month Before Expiry, 33 months).

\begin{tabular}{|c|c|c|c|c|c|c|c|c|c|}
\hline B\# & pH & $\mathbf{A l}^{+++}$Content & $\begin{array}{c}\text { Formaldehyde } \\
\text { Content }\end{array}$ & Thiomersal & $\begin{array}{c}\text { Sterility } \\
\text { Test }\end{array}$ & $\begin{array}{c}\text { Ab. Toxic- } \\
\text { ity }\end{array}$ & $\begin{array}{c}\text { Antigen } \\
\text { Content }\end{array}$ & $\begin{array}{c}\text { Bacterial } \\
\text { Endotoxin }\end{array}$ & $\begin{array}{c}\text { InVivo } \\
\text { Potency }\end{array}$ \\
\hline 510 & 6.28 & $0.351 \mathrm{mg} /$ dose & LT $0.02 \%$ & $0.0094 \%$ & Pass & Pass & $10 \mathrm{Lf} /$ Dose & $0.199 \mathrm{EU} / \mathrm{ml}$ & $75.99 \mathrm{IU} / \mathrm{Dose}$ \\
\hline 511 & 6.55 & $0.269 \mathrm{mg} /$ dose & LT $0.02 \%$ & $0.0090 \%$ & Pass & Pass & $10 \mathrm{Lf} /$ Dose & $0.211 \mathrm{EU} / \mathrm{ml}$ & $74.86 \mathrm{IU} / \mathrm{Dose}$ \\
\hline 512 & 6.35 & $0.253 \mathrm{mg} /$ dose & LT $0.02 \%$ & $0.0089 \%$ & Pass & Pass & $10 \mathrm{Lf} / \mathrm{Dose}$ & $0.240 \mathrm{EU} / \mathrm{ml}$ & $70.52 \mathrm{IU} / \mathrm{Dose}$ \\
\hline 513 & 6.08 & $0.248 \mathrm{mg} /$ dose & LT $0.02 \%$ & $0.0092 \%$ & Pass & Pass & $10 \mathrm{Lf} /$ Dose & $0.239 \mathrm{EU} / \mathrm{ml}$ & $75.22 \mathrm{IU} / \mathrm{Dose}$ \\
\hline 514 & 6.35 & $0.253 \mathrm{mg} /$ dose & LT $0.02 \%$ & $0.0090 \%$ & Pass & Pass & $10 \mathrm{Lf} /$ Dose & $0.180 \mathrm{EU} / \mathrm{ml}$ & $75.14 \mathrm{IU} / \mathrm{Dose}$ \\
\hline
\end{tabular}

Table 4. Stability Data of Different RTF Bulk Filled TT Batches (At expiry month, 36 Months).

\begin{tabular}{|c|c|c|c|c|c|c|c|c|c|}
\hline B\# & pH & $\mathbf{A l}^{+++}$Content & $\begin{array}{c}\text { Formaldehyde } \\
\text { Content }\end{array}$ & Thiomersal & $\begin{array}{c}\text { Sterility } \\
\text { Test }\end{array}$ & $\begin{array}{c}\text { Ab. } \\
\text { Toxicity }\end{array}$ & $\begin{array}{c}\text { Antigen } \\
\text { Content }\end{array}$ & $\begin{array}{c}\text { Bacterial } \\
\text { Endotoxin }\end{array}$ & $\begin{array}{c}\text { InVivo } \\
\text { Potency }\end{array}$ \\
\hline 510 & 6.30 & $0.351 \mathrm{mg} /$ dose & LT $0.02 \%$ & $0.0091 \%$ & Pass & Pass & $10 \mathrm{Lf} / \mathrm{Dose}$ & $0.190 \mathrm{EU} / \mathrm{ml}$ & $62.50 \mathrm{IU} / \mathrm{Dose}$ \\
\hline 511 & 6.39 & $0.279 \mathrm{mg} /$ dose & LT $0.02 \%$ & $0.0090 \%$ & Pass & Pass & $10 \mathrm{Lf} / \mathrm{Dose}$ & $0.222 \mathrm{EU} / \mathrm{ml}$ & $66.41 \mathrm{IU} / \mathrm{Dose}$ \\
\hline 512 & 6.52 & $0.267 \mathrm{mg} /$ dose & LT $0.02 \%$ & $0.0094 \%$ & Pass & Pass & $10 \mathrm{Lf} / \mathrm{Dose}$ & $0.235 \mathrm{EU} / \mathrm{ml}$ & $66.19 \mathrm{IU} / \mathrm{Dose}$ \\
\hline 513 & 6.66 & $0.278 \mathrm{mg} /$ dose & LT $0.02 \%$ & $0.0093 \%$ & Pass & Pass & $10 \mathrm{Lf} / \mathrm{Dose}$ & $0.240 \mathrm{EU} / \mathrm{ml}$ & $66.19 \mathrm{IU} / \mathrm{Dose}$ \\
\hline 514 & 6.62 & $0.278 \mathrm{mg} /$ dose & LT $0.02 \%$ & $0.0094 \%$ & Pass & Pass & $10 \mathrm{Lf} / \mathrm{Dose}$ & $0.170 \mathrm{EU} / \mathrm{ml}$ & $70.39 \mathrm{IU} / \mathrm{Dose}$ \\
\hline
\end{tabular}


Table 5. Stability Data of Different RTF Bulk Filled TT Batches (03 month After Expiry, 39 Months).

\begin{tabular}{|c|c|c|c|c|c|c|c|c|c|}
\hline B\# & $\mathbf{p H}$ & $\mathbf{A l}^{+++}$Content & $\begin{array}{c}\text { Formaldehyde } \\
\text { Content }\end{array}$ & Thiomersal & $\begin{array}{c}\text { Sterility } \\
\text { Test }\end{array}$ & $\begin{array}{c}\text { Ab. } \\
\text { Toxicity }\end{array}$ & $\begin{array}{c}\text { Antigen } \\
\text { Content }\end{array}$ & $\begin{array}{c}\text { Bacterial } \\
\text { Endotoxin }\end{array}$ & $\begin{array}{c}\text { InVivo } \\
\text { Potency }\end{array}$ \\
\hline 510 & 6.72 & $0.350 \mathrm{mg} /$ dose & LT $0.02 \%$ & $0.0092 \%$ & Pass & Pass & $10 \mathrm{Lf} / \mathrm{Dose}$ & $0.195 \mathrm{EU} / \mathrm{ml}$ & $62.50 \mathrm{IU} / \mathrm{Dose}$ \\
\hline 511 & 6.51 & $0.287 \mathrm{mg} /$ dose & LT $0.02 \%$ & $0.0094 \%$ & Pass & Pass & $10 \mathrm{Lf} / \mathrm{Dose}$ & $0.220 \mathrm{EU} / \mathrm{ml}$ & $62.50 \mathrm{IU} / \mathrm{Dose}$ \\
\hline 512 & 6.41 & $0.261 \mathrm{mg} /$ dose & LT $0.02 \%$ & $0.0093 \%$ & Pass & Pass & $10 \mathrm{Lf} / \mathrm{Dose}$ & $0.239 \mathrm{EU} / \mathrm{ml}$ & $62.01 \mathrm{IU} / \mathrm{Dose}$ \\
\hline 513 & 6.50 & $0.271 \mathrm{mg} /$ dose & LT $0.02 \%$ & $0.0093 \%$ & Pass & Pass & $10 \mathrm{Lf} / \mathrm{Dose}$ & $0.235 \mathrm{EU} / \mathrm{ml}$ & $62.01 \mathrm{IU} / \mathrm{Dose}$ \\
\hline 514 & 6.63 & $0.261 \mathrm{mg} /$ dose & LT $0.02 \%$ & $0.0093 \%$ & Pass & Pass & $10 \mathrm{Lf} / \mathrm{Dose}$ & $0.177 \mathrm{EU} / \mathrm{ml}$ & $66.27 \mathrm{IU} / \mathrm{Dose}$ \\
\hline
\end{tabular}

Table 6. Initial Test Data of Formulated TT Bulk Filled Batches.

\begin{tabular}{|c|c|c|c|c|c|c|c|c|c|}
\hline B\# & $\mathbf{p H}$ & $\mathbf{A l}^{+++}$Content & $\begin{array}{c}\text { Formaldehyde } \\
\text { Content }\end{array}$ & Thiomersal & $\begin{array}{c}\text { Sterility } \\
\text { Test }\end{array}$ & $\begin{array}{c}\text { Ab. } \\
\text { Toxicity }\end{array}$ & $\begin{array}{c}\text { Antigen } \\
\text { Content }\end{array}$ & $\begin{array}{c}\text { Bacterial } \\
\text { Endotoxin }\end{array}$ & $\begin{array}{c}\text { InVivo } \\
\text { Potency }\end{array}$ \\
\hline 568 & 6.53 & $0.320 \mathrm{mg} /$ dose & LT $0.02 \%$ & $0.0093 \%$ & Pass & Pass & $10 \mathrm{Lf} / \mathrm{Dose}$ & $0.234 \mathrm{EU} / \mathrm{ml}$ & $74.56 \mathrm{IU} / \mathrm{Dose}$ \\
\hline 569 & 6.22 & $0.300 \mathrm{mg} /$ dose & LT $0.02 \%$ & $0.0094 \%$ & Pass & Pass & $10 \mathrm{Lf} / \mathrm{Dose}$ & $0.227 \mathrm{EU} / \mathrm{ml}$ & $70.08 \mathrm{IU} / \mathrm{Dose}$ \\
\hline 570 & 6.30 & $0.320 \mathrm{mg} /$ dose & LT $0.02 \%$ & $0.0091 \%$ & Pass & Pass & $10 \mathrm{Lf} / \mathrm{Dose}$ & $0.218 \mathrm{EU} / \mathrm{ml}$ & $70.51 \mathrm{IU} / \mathrm{Dose}$ \\
\hline 571 & 6.61 & $0.300 \mathrm{mg} /$ dose & LT $0.02 \%$ & $0.0094 \%$ & Pass & Pass & $10 \mathrm{Lf} / \mathrm{Dose}$ & $0.249 \mathrm{EU} / \mathrm{ml}$ & $74.85 \mathrm{IU} / \mathrm{Dose}$ \\
\hline 572 & 6.64 & $0.300 \mathrm{mg} /$ dose & LT $0.02 \%$ & $0.0091 \%$ & Pass & Pass & $10 \mathrm{Lf} / \mathrm{Dose}$ & $0.231 \mathrm{EU} / \mathrm{ml}$ & $79.31 \mathrm{IU} / \mathrm{Dose}$ \\
\hline Specs & $\mathbf{6 - 7}$ & NMT $1.25 \mathbf{m g} /$ dose & $\begin{array}{c}\text { NMT } \\
\mathbf{0 . 0 2} \%\end{array}$ & $\begin{array}{c}\mathbf{0 . 0 0 8 5} \%- \\
\mathbf{0 . 0 1 1 5} \%\end{array}$ & Sterile & Non-toxic & $\begin{array}{c}\mathbf{5 - 2 5} \mathbf{L f} / \\
\text { dose }\end{array}$ & NMT 25 EU/ml & NLT 40 IU/Dose \\
\hline
\end{tabular}

Manuf. Date; Jan. 2012

Expiry Date; Dec. 2014

Table 7. Stability Data of Local Formulated Bulk Filled Batches (06 month Before Expiry, 30 Months).

\begin{tabular}{|c|c|c|c|c|c|c|c|c|c|}
\hline B\# & $\mathbf{p H}$ & $\mathbf{A l}^{+++}$Content & $\begin{array}{c}\text { Formaldehyde } \\
\text { Content }\end{array}$ & Thiomersal & $\begin{array}{c}\text { Sterility } \\
\text { Test }\end{array}$ & $\begin{array}{c}\text { Ab. } \\
\text { Toxicity }\end{array}$ & $\begin{array}{c}\text { Antigen } \\
\text { Content }\end{array}$ & $\begin{array}{c}\text { Bacterial } \\
\text { Endotoxin }\end{array}$ & $\begin{array}{c}\text { InVivo } \\
\text { Potency }\end{array}$ \\
\hline 568 & 6.58 & $0.320 \mathrm{mg} /$ dose & LT $0.02 \%$ & $0.0092 \%$ & Pass & Pass & $10 \mathrm{Lf} / \mathrm{Dose}$ & $0.237 \mathrm{EU} / \mathrm{ml}$ & $66.19 \mathrm{IU} / \mathrm{Dose}$ \\
\hline 569 & 6.46 & $0.320 \mathrm{mg} /$ dose & LT $0.02 \%$ & $0.0094 \%$ & Pass & Pass & $10 \mathrm{Lf} / \mathrm{Dose}$ & $0.230 \mathrm{EU} / \mathrm{ml}$ & $66.42 \mathrm{IU} / \mathrm{Dose}$ \\
\hline 570 & 6.53 & $0.297 \mathrm{mg} /$ dose & LT $0.02 \%$ & $0.0095 \%$ & Pass & Pass & $10 \mathrm{Lf} / \mathrm{Dose}$ & $0.221 \mathrm{EU} / \mathrm{ml}$ & $62.50 \mathrm{IU} / \mathrm{Dose}$ \\
\hline 571 & 6.33 & $0.324 \mathrm{mg} /$ dose & LT $0.02 \%$ & $0.0093 \%$ & Pass & Pass & $10 \mathrm{Lf} / \mathrm{Dose}$ & $0.253 \mathrm{EU} / \mathrm{ml}$ & $62.50 \mathrm{IU} / \mathrm{Dose}$ \\
\hline 572 & 6.38 & $0.380 \mathrm{mg} /$ dose & LT $0.02 \%$ & $0.0093 \%$ & Pass & Pass & $10 \mathrm{Lf} / \mathrm{Dose}$ & $0.235 \mathrm{EU} / \mathrm{ml}$ & $66.14 \mathrm{IU} / \mathrm{Dose}$ \\
\hline
\end{tabular}

Table 8. Stability Data of Local Formulated Bulk Filled Batches (03 month Before Expiry, 33 Months).

\begin{tabular}{|c|c|c|c|c|c|c|c|c|c|}
\hline B\# & $\mathbf{p H}$ & $\mathbf{A l}^{+++}$Content & $\begin{array}{c}\text { Formaldehyde } \\
\text { Content }\end{array}$ & Thiomersal & $\begin{array}{c}\text { Sterility } \\
\text { Test }\end{array}$ & $\begin{array}{c}\text { Ab. } \\
\text { Toxicity }\end{array}$ & $\begin{array}{c}\text { Antigen } \\
\text { Content }\end{array}$ & $\begin{array}{c}\text { Bacterial } \\
\text { Endotoxin }\end{array}$ & $\begin{array}{c}\text { InVivo } \\
\text { Potency }\end{array}$ \\
\hline 568 & 6.30 & $0.320 \mathrm{mg} /$ dose & LT $0.02 \%$ & $0.0091 \%$ & Pass & Pass & $10 \mathrm{Lf} / \mathrm{Dose}$ & $0.233 \mathrm{EU} / \mathrm{ml}$ & $63.72 \mathrm{IU} / \mathrm{Dose}$ \\
\hline 569 & 6.39 & $0.320 \mathrm{mg} /$ dose & LT $0.02 \%$ & $0.0091 \%$ & Pass & Pass & $10 \mathrm{Lf} / \mathrm{Dose}$ & $0.228 \mathrm{EU} / \mathrm{ml}$ & $63.84 \mathrm{IU} / \mathrm{Dose}$ \\
\hline 570 & 6.51 & $0.297 \mathrm{mg} /$ dose & LT $0.02 \%$ & $0.0093 \%$ & Pass & Pass & $10 \mathrm{Lf} / \mathrm{Dose}$ & $0.219 \mathrm{EU} / \mathrm{ml}$ & $60.23 \mathrm{IU} / \mathrm{Dose}$ \\
\hline 571 & 6.34 & $0.320 \mathrm{mg} /$ dose & LT $0.02 \%$ & $0.0094 \%$ & Pass & Pass & $10 \mathrm{Lf} / \mathrm{Dose}$ & $0.251 \mathrm{EU} / \mathrm{ml}$ & $66.02 \mathrm{IU} / \mathrm{Dose}$ \\
\hline 572 & 6.25 & $0.393 \mathrm{mg} /$ dose & LT $0.02 \%$ & $0.0089 \%$ & Pass & Pass & $10 \mathrm{Lf} / \mathrm{Dose}$ & $0.232 \mathrm{EU} / \mathrm{ml}$ & $66.02 \mathrm{IU} / \mathrm{Dose}$ \\
\hline
\end{tabular}

Table 9. Stability Data of Local Formulated Bulk Filled Batches (At expiry month, 36 Months).

\begin{tabular}{|c|c|c|c|c|c|c|c|c|c|}
\hline B\# & $\mathbf{p H}$ & $\mathbf{A l}^{++}$Content & $\begin{array}{c}\text { Formaldehyde } \\
\text { Content }\end{array}$ & Thiomersal & $\begin{array}{c}\text { Sterility } \\
\text { Test }\end{array}$ & $\begin{array}{c}\text { Ab. } \\
\text { Toxicity }\end{array}$ & $\begin{array}{c}\text { Antigen } \\
\text { Content }\end{array}$ & $\begin{array}{c}\text { Bacterial } \\
\text { Endotoxin }\end{array}$ & $\begin{array}{c}\text { InVivo } \\
\text { Potency }\end{array}$ \\
\hline 568 & 6.26 & $0.296 \mathrm{mg} /$ dose & LT $0.02 \%$ & $0.0091 \%$ & Pass & Pass & $10 \mathrm{Lf} /$ Dose & $0.231 \mathrm{EU} / \mathrm{ml}$ & $61.25 \mathrm{IU} / \mathrm{Dose}$ \\
\hline 569 & 6.49 & $0.323 \mathrm{mg} /$ dose & LT $0.02 \%$ & $0.0090 \%$ & Pass & Pass & $10 \mathrm{Lf} /$ Dose & $0.221 \mathrm{EU} / \mathrm{ml}$ & $61.26 \mathrm{IU} / \mathrm{Dose}$ \\
\hline 570 & 6.51 & $0.270 \mathrm{mg} /$ dose & LT $0.02 \%$ & $0.0091 \%$ & Pass & Pass & $10 \mathrm{Lf} / \mathrm{Dose}$ & $0.222 \mathrm{EU} / \mathrm{ml}$ & $57.97 \mathrm{IU} / \mathrm{Dose}$ \\
\hline 571 & 6.41 & $0.324 \mathrm{mg} /$ dose & LT $0.02 \%$ & $0.0094 \%$ & Pass & Pass & $10 \mathrm{Lf} /$ Dose & $0.250 \mathrm{EU} / \mathrm{ml}$ & $61.71 \mathrm{IU} / \mathrm{Dose}$ \\
\hline 572 & 6.30 & $0.380 \mathrm{mg} /$ dose & LT $0.02 \%$ & $0.0091 \%$ & Pass & Pass & $10 \mathrm{Lf} /$ Dose & $0.230 \mathrm{EU} / \mathrm{ml}$ & $65.90 \mathrm{IU} / \mathrm{Dose}$ \\
\hline
\end{tabular}


Table 10. Stability Data of Local Formulated Bulk Filled Batches (03 month After Expiry, 39 Months).

\begin{tabular}{|c|c|c|c|c|c|c|c|c|c|}
\hline B\# & $\mathbf{p H}$ & $\mathbf{A l}^{+++}$Content & $\begin{array}{c}\text { Formaldehyde } \\
\text { Content }\end{array}$ & Thiomersal & $\begin{array}{c}\text { Sterility } \\
\text { Test }\end{array}$ & $\begin{array}{c}\text { Ab. } \\
\text { Toxicity }\end{array}$ & $\begin{array}{c}\text { Antigen } \\
\text { Content }\end{array}$ & $\begin{array}{c}\text { Bacterial } \\
\text { Endotoxin }\end{array}$ & $\begin{array}{c}\text { InVivo } \\
\text { Potency }\end{array}$ \\
\hline 568 & 6.41 & $0.355 \mathrm{mg} /$ dose & LT $0.02 \%$ & $0.0092 \%$ & Pass & Pass & $10 \mathrm{Lf} / \mathrm{Dose}$ & $0.234 \mathrm{EU} / \mathrm{ml}$ & $58.58 \mathrm{IU} / \mathrm{Dose}$ \\
\hline 569 & 6.43 & $0.323 \mathrm{mg} /$ dose & LT $0.02 \%$ & $0.0089 \%$ & Pass & Pass & $10 \mathrm{Lf} / \mathrm{Dose}$ & $0.226 \mathrm{EU} / \mathrm{ml}$ & $57.86 \mathrm{IU} / \mathrm{Dose}$ \\
\hline 570 & 6.29 & $0.351 \mathrm{mg} /$ dose & LT $0.02 \%$ & $0.0090 \%$ & Pass & Pass & $10 \mathrm{Lf} / \mathrm{Dose}$ & $0.217 \mathrm{EU} / \mathrm{ml}$ & $55.59 \mathrm{IU} / \mathrm{Dose}$ \\
\hline 571 & 6.39 & $0.324 \mathrm{mg} /$ dose & LT $0.02 \%$ & $0.0091 \%$ & Pass & Pass & $10 \mathrm{Lf} / \mathrm{Dose}$ & $0.251 \mathrm{EU} / \mathrm{ml}$ & $57.70 \mathrm{IU} / \mathrm{Dose}$ \\
\hline 572 & 6.31 & $0.380 \mathrm{mg} /$ dose & LT $0.02 \%$ & $0.0093 \%$ & Pass & Pass & $10 \mathrm{Lf} / \mathrm{Dose}$ & $0.237 \mathrm{EU} / \mathrm{ml}$ & $61.71 \mathrm{IU} / \mathrm{Dose}$ \\
\hline
\end{tabular}

Figure 1. InVivo Potency Test Trend of T.T injection 0.5ml (RTF) near and after expiry.

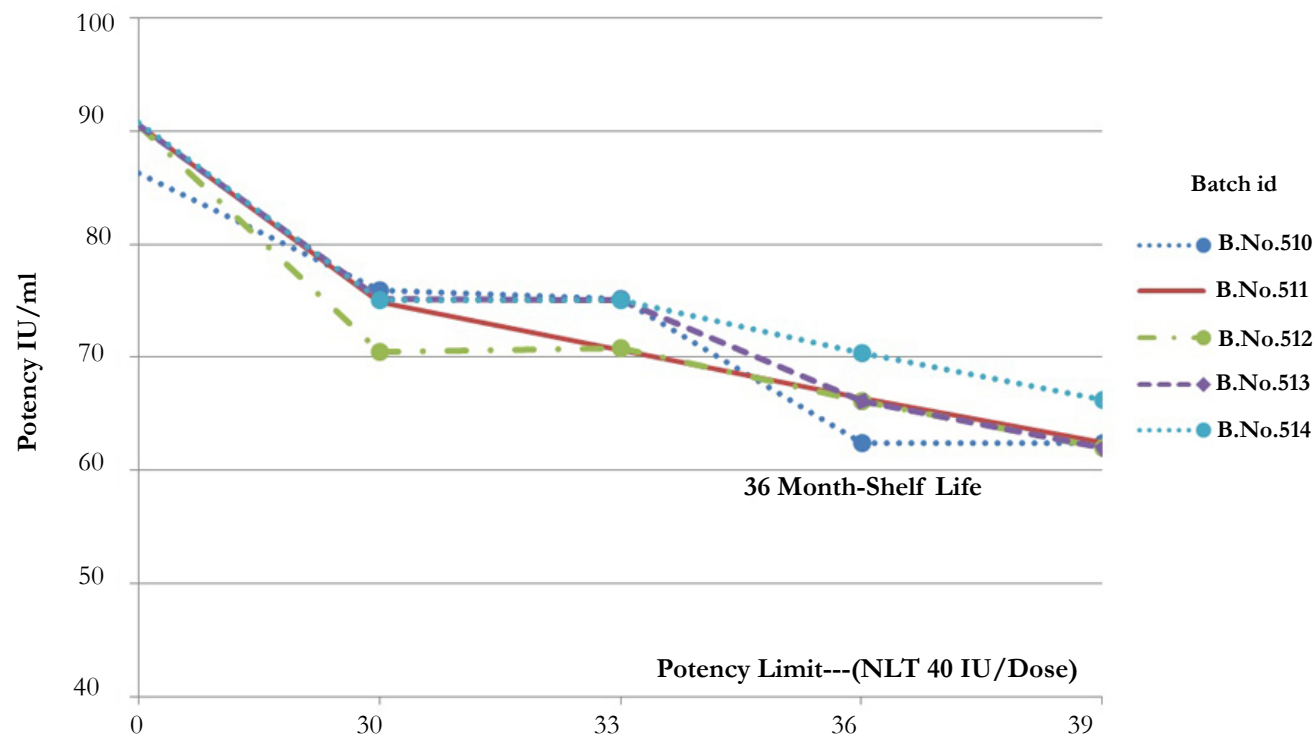

Time Period (Month)

Figure 2. InVivo Potency Trend of Local Formulated T.T Injection 0.5ml near and after expiry.

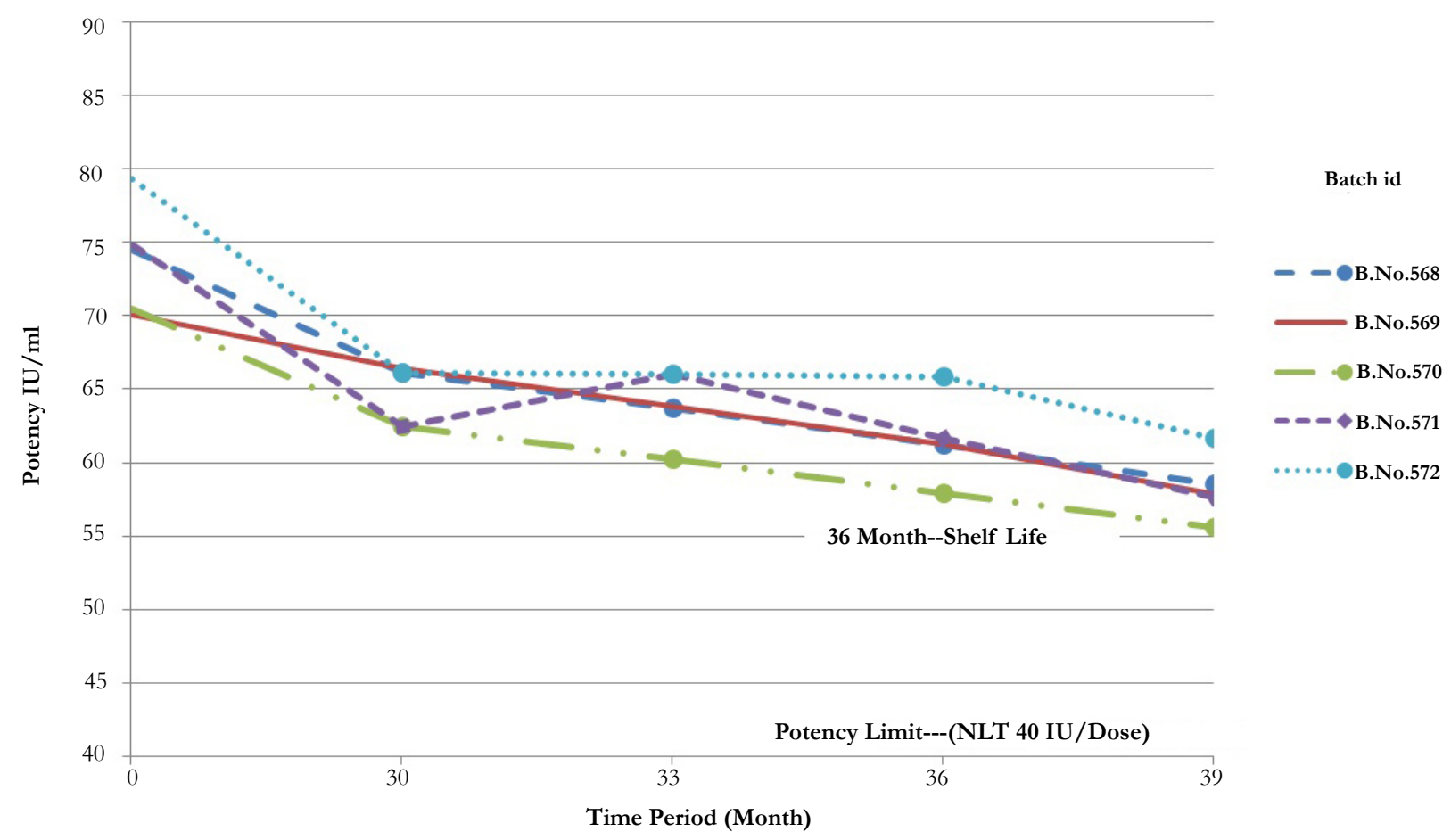

Tetanus Toxoid vaccine RTF Bulk is showing maximum decrease of $0.73 \mathrm{IU} /$ dose/month that means less than 1.0 unit decrease in InVivo potency per dose per month even three months after its expiry. 
Figure 3. Decrease in Potency per month of T.T batches near and after expiry.

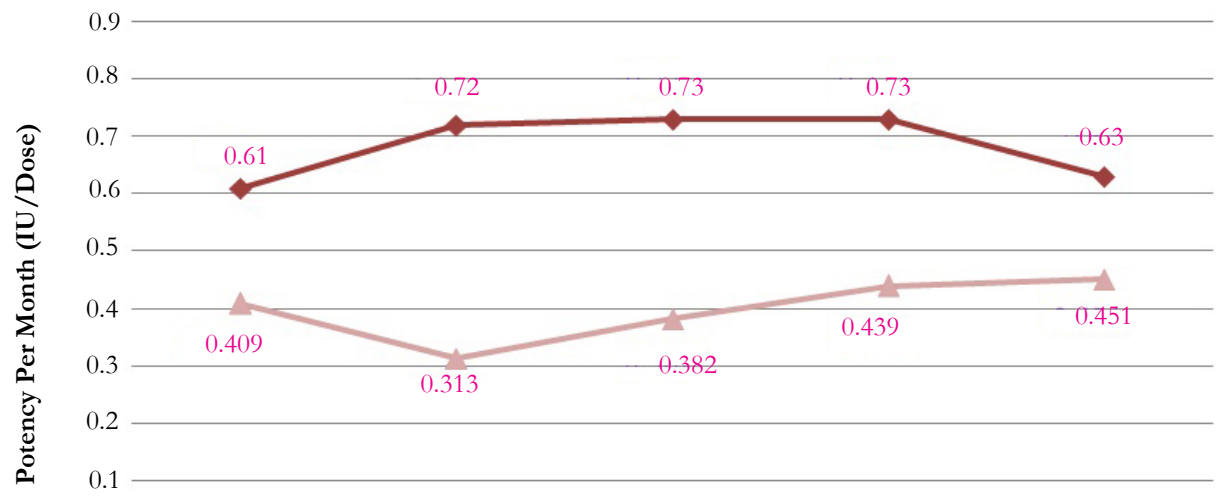

0

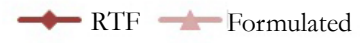

Tetanus Toxoid vaccine Formulated Bulk is showing less than 0.451 unit decrease in InVivo potency IU/dose/month that means less than 0.5 unit decrease in InVivo potency per dose per month even three months after its expiry.

formaldehyde content after 39 months of formulated come up within limit of LT $0.02 \% . \mathrm{Al}^{+++}$content is reported $0.320 \mathrm{mg} /$ dose, $0.300 \mathrm{mg} /$ dose, $0.320 \mathrm{mg} /$ dose, $0.300 \mathrm{mg} / \mathrm{dose}, 0.300 \mathrm{mg} /$ dose for batch 1,2,3,4 and 5 respectively. $\mathrm{pH}$ of batches vaccines exhibited within limits i-e 6-7 after 39 months.

Tetanus Toxoid formulated at private sector facility fulfill all the standards set by WHO i.e, Sterility, Antigenic content, Bacterial Endotoxin along with safety profile. It can be concluded that formulated vaccine prevail its efficacy and safe for human use.

\section{Discussion}

Vaccines are administered within a specific range of potency that is established through undeniable testing studies. Vaccines loose their efficacy with the passage of time in term of potency [11, 12]. World Health Organization (WHO) recommends adequate stability studies for the production and Quality control of vaccines as an essential part of vaccine development [9]. The data on retained vaccine potency at recommended refrigerated storage temperature are clinically useful and always be maintained. Further studies at different conditions may be useful in determining the behavior of vaccine during short term accidental exposure to accelerated conditions, such as during transport, which can compromise product quality.

Our comparative data collected from ten different batches of vaccines (RTF \& Local formulated) shelf-life conditions as mentioned above in results, showing assurance and physical revelation of vaccines. Moreover the free formaldehyde content, $\mathrm{Al}^{+++}$content, thiomersal concentration and $\mathrm{pH}$ of vaccines remained within range for shelf-life period, were found to conform the WHO's limits. Real time stability studies also play a important role in assuring product quality at all points in the vaccine life cycle. The stability studies on product quality especially potency provide a critical link between marketed and safety profile evaluated vaccine product, addressing important regulatory concerns by assuring that product quality is maintained throughout the declared shelf life period. During development, stability studies are done to assure product quality and to obtain the data needed to support licensing.
Our studies showed no degradation or loss of potency below limit during recommended storage temperature and studies shows the stability of the formulation. The loss of potency of the vaccine below limit can leads towards the occurance of neonatal tetanus. The determinants of the incidence of neonatal tetanus also relate to the cultural diversity of unhygienic childbirth practices and cord care $[4,13]$ as well. The unskilled attendance with delivery, parent's illiteracy, lack of antenatal care, including low level or no immunization against tetanus, geographical location and climatic prevalence of spores of Clostridium tetani and rural settled populations [7] are some of the factors responsible for this deadly disease. Vaccine shelf-life must be best managed through determination of a minimum potency release requirement, which helps assure good potency throughout expiry.In our study, the testing of the vaccines has been done at the time of batch manufacturing, then six months before expiry, three months before expiry, at expiry month and has also extended its testing for another three months after its expiry for both RTF \& Local Formulated bulk filled batches.

The results were found to be very satisfactory. It shows that locally formulated bulk is equally effective as compared with the imported Tetanus Toxoid bulk and stability program also helps assure continued quality of product throughout the shelf life period, while comparability studies are performed for different process or facilities change in order to demonstrate that the change play some role but has not impacted the stability characteristics of the product. Evaluation of stability is an essential part of the assessment of the vaccine quality. Indeed, the stability studies are aimed at verifying that the vaccines maintain their efficacy/ quality criteria throughout their shelf lives [14]. Worth mentioning that excursions from storage condition requirements may affect product performance and stability. The effects of temperature on stability depend on the period of time that a product is subjected to these conditions, temperature level and activation energy. The effects of excursions on shelf life performance are less severe when lower level of risk is implemented to establish the claimed shelf life.

Although local manufacturer unlike manufacturers in India 
produces small bulk batches in 20Lit. bottles and each bulk bottle is filled in a separate finish batch ID yet the findings of our local formulated tetanus vaccine studies carried out at Amson Vaccines \& Pharma (Pvt) Ltd., Islamabad, Pakistan are quite comparable to those with imported tetanus vaccine produced in 1000Lit. of bulk batch container. The Impact of these studies will be in the promotion of locally formulated vaccine usage and help in saving millions of dollars required for the purchase and import of vaccines and will reduce the financial burden on national manufacturers. These studies also address regarding quality, efficacy and potency of vaccines produced by Private Sector (Amson Vaccines \& Pharma (Pvt) Ltd, Islamabad) of Pakistan. The scope of these studies also includes a positive impact on reducing neonatal deaths because of this preventable disease and promotion of Expanded Program of Immunization in Pakistan.

Although initial InVivo potency in case of RTF bulk is better yet loss in potency per dose per month is better in case of local formulated bulk, that may be due to quality of Aluminum Phosphate gel and slow release of antigen from the surface of the gel. This reduced rate in potency of locally formulated bulk indicates that bulk formulated at private sector has some significance over the RTF bulk imported from the principal.

The present findings also indicate stability and provide declaration that this tetanus vaccine can remain effective even at least three months after its expiry if stored at recommended temperature under routine shelf life and use when suggested measures for storage and handling are followed in true spirit.

\section{Acknowledgement}

Thanks to Shahzad Abbas, Muhammad Aslam and Muhammad Jamil for their assistance.

\section{References}

[1]. Vandelaer JBM, Gasse F, Kurian M, Shaw C,Garnier S (2003) Tetanus in developing countries: An update on the Maternal and Neonatal Tetanus Elimination Initiative. Vaccine 21(24): 3442-3445.

[2]. Tierney RNT, Parkins CJ, Caposio P, Fairweather NF, Sesardic D et al. (2012) A single-dose cytomegalovirus-based vaccine encoding tetanus toxin fragment $\mathrm{C}$ induces sustained levels of protective tetanus toxin antibodies in mice. Vaccine 30(20): 3047-3052.

[3]. Fikree FF, ASa BH (2002) Time to focus child survival programmes on the newborn: Assessment of levels and causes of infant mortality in rural Pakistan. Bull World Health Organ 80(4): 271-276.

[4]. Quddus ALS, Rahbar M,Pervaiz Y (2002) Neonatal tetanus: Mortality rate and risk factors in Loralai District Pakistan. Int. J. Epidemiol 31(3): 648653.

[5]. WHO (2015) Maternal and Neonatal Tetanus (MNT) elimination Initiative.http://www.who.int/immunization/diseases/MNTE_initiative/en/index.html

[6]. WHO (2015) Selected Infectious Diseases. World Health Statistics http:// www.who.int/immunization/monitoring_surveillance/en/index.html.

[7]. Parveen GHS, Malik F, Begum A. Mahmood S, Wajid A, Raja FY, et al. (2012) Seroepidemiological surveillance of antitetanus antibodies in Pakistani childbearing women: A population based survey. Afr. J. Pharm. Pharmacol 6(36): 2621-2628.

[8]. Plotkin S, Orenstein W, Offit P (2008) A short history of vaccination. Vaccines. (5th edn), W.B. Saunders company, Philadelphia . 805-840.

[9]. Knezevic I (2009) Stability evaluation of vaccines: WHO approach. Biologicals 37(6): 357-359.

[10]. WHO (2014) Guidelines on stability evaluation of vaccines. WHO Expert Committee on Biological Standardization, Sixty-third report, Technical Report Series No 980, Geneva.

[11]. Jodar L, GEa FI (2004) Scientific challenges for the quality control and production of group C meningococcal conjugate vaccines. Vaccine 22(8): 1047-1053.

[12]. TET aS (2009) Basic principles of stability. Biologicals 37(6): 379-386.

[13]. Bennett J, Schooley M, Traverso H, Agha SB, Boring J (1996) Bundling: A newly identified risk factor for neonatal tetanus: implications for global control. Int J Epidemiol 25(4): 879-884.

[14]. Socarras S, Magari RT (2009) Modeling the effects of storage temperature excursions on shelf life. J Pharm Biomed Anal 49(2): 221-226. 